\title{
Real-time Resource Positioning System based on Wireless Sensor Network in Manufacturing Workshop
}

\author{
https://doi.org/10.3991/ijoe.v13i06.6933 \\ Shaobo Li \\ Chinese Academy of Sciences, Chengdu, China \\ Guizhou Unversity, Guiyang, China \\ lishaobo@gzu.edu.cn \\ Jinglei Qu \\ Chinese Academy of Sciences, Chengdu, China \\ jinglei0526@163.com \\ Chenglong Zhang \\ Guizhou Unversity, Guiyang, China \\ 995010174 @qq. com
}

\begin{abstract}
Discrete manufacturing enterprise has a complex and varied production process, resulting in the dynamic characteristics of manufacturing resources. Aiming at the efficient collection and management of manufacturing resource information, improving the intellectualization of enterprises with a real-time resource positioning system based on wireless sensor network was proposed. First, a perceptual model for resource positioning, which can collect and analyze real-time resource information in the workshop, was designed. Second, the architectural structure of a real-time resource positioning system was designed based on wireless sensor network, and the resource positioning flow was illustrated. Third, a multi-sensor positioning data fusion algorithm based on fuzzy evidence theory was proposed to address the low positioning accuracy caused by the electromagnetic interference and obstacle in the manufacturing workshop environment. Finally, a prototype system was implemented to demonstrate the validity of the method in practice.
\end{abstract}

Keywords-resource positioning, wireless sensor network, intelligent workshop, internet of manufacturing things

\section{$1 \quad$ Introduction}

The Internet of Manufacturing Things (IoMT) is a novel manufacturing model that deeply integrates advanced manufacturing technology with electronic information and intelligent sensor technologies; this integration is conducive for promoting the intellectualization of the manufacturing process [1-3]. Discrete manufacturing enterprise has various product specifications, complicated production processes, and numerous suppliers and customers. Manufacturing resources such as raw materials, equipment, 
tools, processing environments, and employees have dynamic characteristics [4,5]. To effectively manage manufacturing resources, ensure production safety, and improve production efficiency, as well as enhance the intelligent level of manufacturing, collecting real-time resource positioning information is necessary.

The global positioning system (GPS) is affected by the constraints of the manufacturing workshop environment and the costs of installation; thus, it fails to effectively solve the positioning problem in discrete manufacturing workshops. With the rapid development of the wireless sensor network (WSN) technology, a low-consumption, low-cost sensor node is applied in many fields [6-9], including smart group network, smart industry, mine check and so on. However, the positioning system in manufacturing workshops has low accuracy because of the influence of electromagnetic interference and obstacle in such environment.

To locate manufacturing resources and to improve the positioning accuracy in workshops, this paper first proposes a perceptual model for resource position. Second, the architectural structure of a real-time resource positioning system is designed based on the perceptual model, and a multi-sensor positioning data fusion algorithm is then presented. Finally, a prototype system is implemented and demonstrated the solution is feasible and efficient.

\section{Perceptual Model for Resource Positioning}

To satisfy the requirement of the workshop resource positioning in discrete manufacturing enterprises, the interconnection between the automatic control equipment and the manufacturing execution systems of the workshop is realized using IoMT technologies.

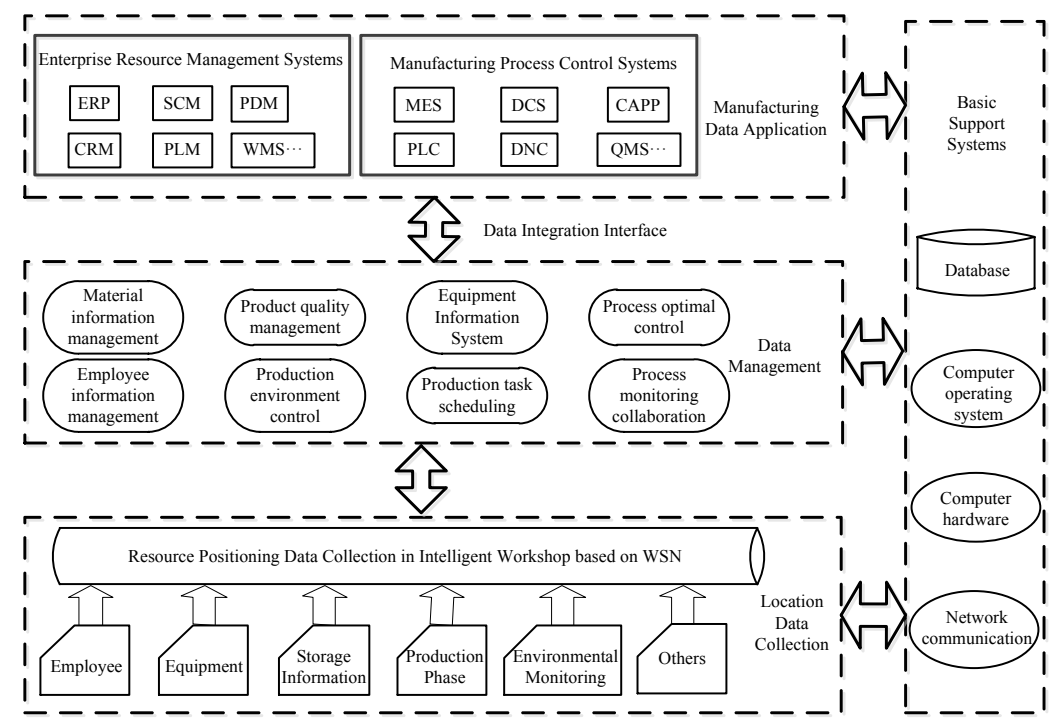

Fig. 1. Perceptual model for resource positioning in manufacturing workshops 
The perceptual model for resource positioning in manufacturing workshops, which is based on the intelligent workshop wireless acquisition terminal, is presented in Fig. 1. According to the matched effective resource tags, the real-time acquisition and analysis of the resource information in the workshop is realized. By improving the integrity of the manufacturing data and strengthening the management and business application of manufacturing information, the proposed model can monitor and analyze the manufacturing process, predict product quality, and provide reference for decision-makers.

\section{System Structure and Principle}

The real-time resource positioning system based on wireless sensor network is mainly composed of hardware and software systems. The hardware system includes tags, readers, uninterruptible power supply, network communication equipment and management servers. This system is used to identify and collect manufacturing resource data, such as employee, material, and storage information. The collected information is transmitted to the location software system for analysis and processing through a wireless communication network. The software system is mainly utilized for data acquisition, processing, display, and storage. Furthermore, the software system can communicate with the enterprise resource management and manufacturing process control systems to transmit data through the data integration interface. Workshop managers can visually obtain the statuses of manufacturing resources through the graphical interface of the software.

The workshop environment has strong electromagnetic interference with widely distributed data collection points. Considering the actual demand, as well as the reliability, real-time capability, and scalability of the system, a real-time resource positioning system was proposed based on WSN. The architectural structure of the system is shown in Fig. 2.

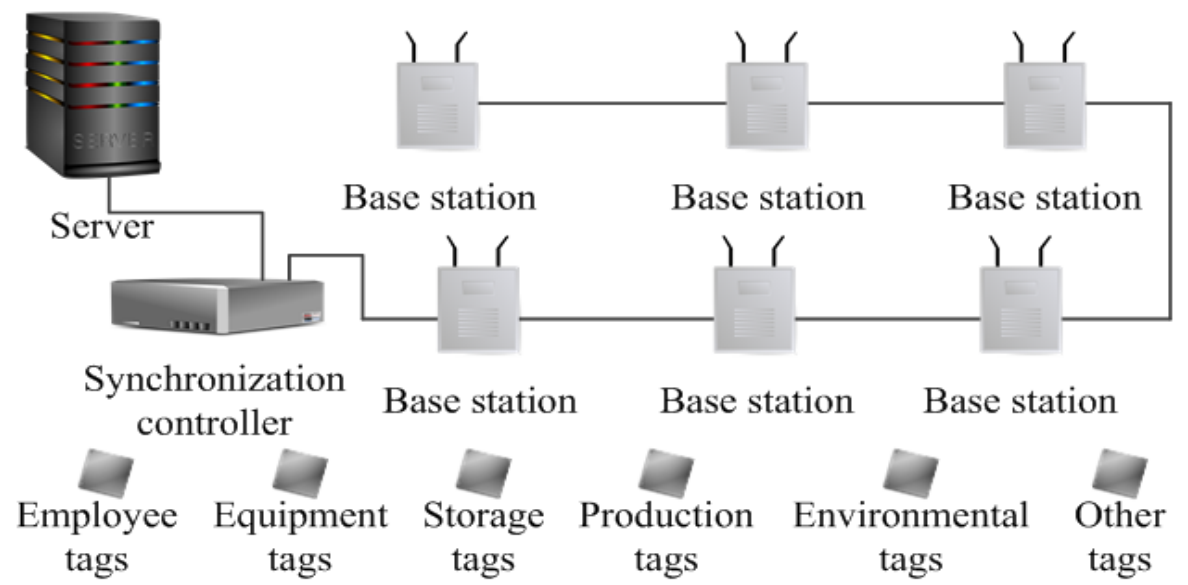

Fig. 2. Architectural structure of the resource positioning system 
A working flow chart of the system is shown in Fig. 3. After entering the positioning network, positioning tags are activated upon receiving the identification signal sent out by the synchronization controller. When the activated tags receive the working signal, it will send out real-time wireless signals. Readers receive the signals from the tags through the antenna, and the signals that arrived at different times are processed to generate the time difference data packets. Data packets are transmitted to the synchronization controller through the network. The positioning server then calculates the position of the tag according to the data packets. The practical application of the measurement data of each sensor is not completely reliable due to interference from the working environment and human factors. A large deviation measurement or even wrong information will affect the accuracy of data fusion and even lead to wrong fusion results. To solve this problem, multi-sensor position data fusion algorithm is designed. According to the measured position values obtained from multiple base stations, the system can improve positioning accuracy by filtering and fusing redundant information among multiple sets of measurements.

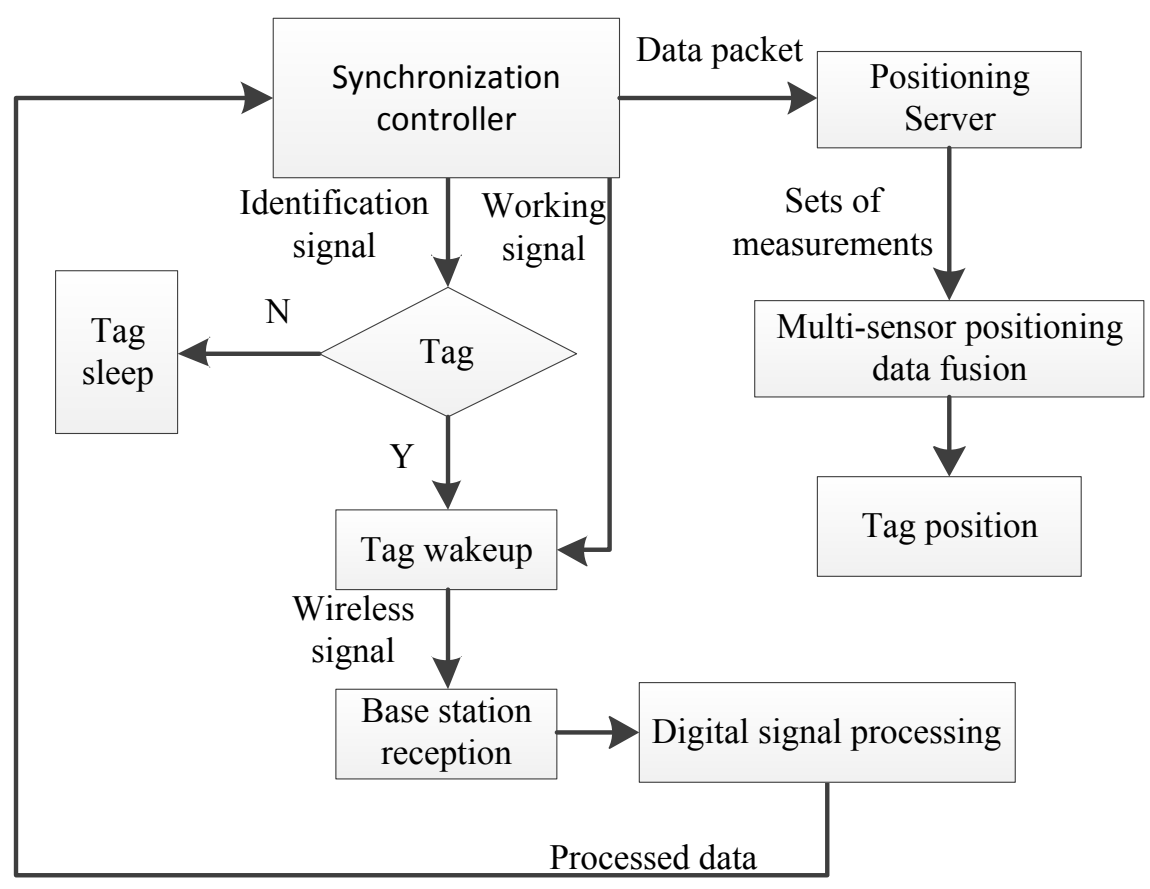

Fig. 3. Resource positioning flow chat

\section{$4 \quad$ Multi-sensor Positioning Data Fusion Algorithm}

The positioning system can simultaneously obtain multiple sets of measurements from the same tag by increasing the number of base stations. To locate a material in the workshop, the real-time measurement data of the location system are $Z_{i}=\left(x_{i}, y_{i}\right)(i$ 
$=1,2, \ldots, n)$. Based on the principle of evidence theory, all measurements $\left\{Z_{1}, Z_{2}, \ldots\right.$, $\left.Z_{n}\right\}$ are used as discernment frame $\Theta$, and then converted into evidence for further combination. Owing to the influence of ambient noise and the accuracy of sensors, the measurements can be regarded as the superposition of the true value and the noise. All the measurements within the normal deviation range are constantly centered on the true value and scattered in the neighborhood. The measurement error of sensors approximation obeys a normal distribution, and we selected the normalized distribution function as the membership function.

$\mu_{i j}$ is defined as the membership degree of $Z_{j}$ to $Z_{i}$ for the measurement $Z_{i}$ in the identification frame.

$$
\mu_{i j}=e^{-\left[\left(x_{i}-x_{j}\right)^{2}+\left(y_{i}-y_{j}\right)^{2}\right]}
$$

The larger the $\mu_{i j}$, the nearer $Z_{j}$ is to $Z_{i}$, which indicates that $Z_{j}$ has a higher degree of support to $Z_{i}$. On the contrary, it has a lower degree of support. To the discernment frame $\Theta$, the membership degree matrix of $n$ measurements for each other is given as:

$$
U=\left[\begin{array}{cccc}
\mu_{11} & \mu_{12} & \mathrm{~L} & \mu_{1 n} \\
\mu_{21} & \mu_{22} & \mathrm{~L} & \mu_{2 n} \\
\mathrm{M} & \mathrm{M} & & \mathrm{M} \\
\mu_{n 1} & \mu_{n 1} & \mathrm{~L} & \mu_{n n}
\end{array}\right]
$$

Membership degree vector $\boldsymbol{p}_{i}(i=1,2, \ldots, n)$ is the $i$-th row of the membership matrix $U, \boldsymbol{p}_{i}=\left(\mu_{i 1}, \mu_{i 2}, \ldots, \mu_{i n}\right)$, which indicates the membership degree vector of $Z_{i}$ to the discernment frame $\Theta$. The length of vector $\left|p_{i}-p_{j}\right|$ is called the Euclidean distance of $\boldsymbol{p}_{i}$ and $\boldsymbol{p}_{j}$, which represents the confidence distance measure of $Z_{i}$ and $Z_{j}$, and it is denoted by:

$$
d_{i j}=\left\|p_{i}-p_{j}\right\|=\sqrt{\sum_{k=1}^{n}\left(\mu_{i k}-\mu_{j k}\right)}
$$

The confidence distance of any two measurements should be symmetric; thus, $d_{i j}=$ $d_{j i}$, and the confidence distance from $Z_{i}$ to $Z_{j}$ is equal to the confidence distance from $Z_{j}$ to $Z_{i}$. The higher the confidence distance value, the greater the difference and the lower the mutual support between $Z_{i}$ and $Z_{j}$.

The mean square Euclidean distance of $Z_{i}$ to all measurements is $s_{i}$, which refers to the difference degree between $Z_{i}$ and other measurements.

$$
s_{i}=\frac{1}{n} \sum_{j=1}^{n} d_{i j}^{2}
$$




$$
\bar{s}=\frac{1}{n} \sum_{i=1}^{n} s_{i}
$$

A smaller $s_{i}$ indicates that $Z_{i}$ has minimal difference with others, which means that $Z_{i}$ has a high degree of reliability. On the contrary, a larger $s_{i}$ represents the singularity of measurement $Z_{i}$; thus, it has low credibility.

Based on the reliability evaluation of the measurements, a basic trust assignment method is proposed to convert $Z_{i}$ to evidence $e_{i}$. If the mean square Euclidean distance of the measurement is too large, then it has a large deviation that should be removed. Otherwise, it is an effective measurement, and the basic trust allocation should meet the following formula:

$$
\left\{\begin{array}{c}
m_{i}\left(Z_{j}\right)=0, s_{j}>\delta \bar{s} \\
\frac{m_{i}\left(Z_{j 1}\right)}{m_{i}\left(Z_{j 2}\right)}=\frac{s_{j 2}}{s_{j 1}}, s_{j} \leq \delta \bar{s}
\end{array}\right.
$$

where $Z_{j 1}$ and $Z_{j 2}$ are any two valid measurements, and $\delta$ is the threshold coefficient. After a number of groups of data fusion experiments, a generally better result is obtained by taking $\delta=1.25$. In the actual operation of the process, (6) is used to obtain the set of confidence coefficients $\left\{\omega_{i}\right\}(i=1,2, \ldots, n)$.

Confidence coefficient is used to normalize and to revise the weight of the membership degree of each measurement. The basic trust assignment is then obtained, and the measurement value is completely transformed in the evidence conversion.

$$
m_{i}\left(Z_{j}\right)=\omega_{j} \mu_{i j} / \sum_{k=1}^{n} \omega_{k} \mu_{i k}
$$

Equation (7) meets the quality of the basic trust distribution function in evidence theory. This method reduces the influence of a large deviation measurement on the fusion by evaluating the reliability of the sensor.

A high degree of conflict between the generated evidence, which leads to the paradox of inference results, may be observed. Thus, the probability of supporting evidence conflict is distributed to each measurement in the evidence combination process using the combination formula (8).

$$
m\left(Z_{i}\right)=\prod_{j=1}^{n} m_{j}\left(Z_{i}\right)+c \bar{m}_{j}\left(Z_{i}\right)
$$

where $c$ is the conflict factor.

$$
c=1-\sum_{i=1}^{n} \prod_{j=1}^{n} m_{j}\left(x_{i}\right)
$$


$\bar{m}_{j}\left(Z_{i}\right)$ is the mean value of basic trust assignment of $Z_{i}$ in the evidence.

$$
\bar{m}_{j}\left(Z_{i}\right)=\frac{1}{n} \sum_{j=1}^{n} m_{j}\left(Z_{i}\right)
$$

The combined evidence $m\left(Z_{i}\right)$ is the weight coefficient of $Z_{i}$. Finally, the position data fusion result is

$$
Z_{0}=\sum_{i=1}^{n} Z_{i} m\left(Z_{i}\right)
$$

\section{System Implementation}

The real-time resource positioning system uses the hierarchical B/S structure design, Visual Studio 2015 as the development platform and $\mathrm{C} \#$ as the programming language. The resource position scenario is constructed based on Geographic Information System. The system development includes the interface design and implementation, the database, and the software functions. The function module is mainly divided into the information management, real-time positioning, management, and resource tracking modules. Parts of the system application interface are shown in Fig. 4, where (a) is the manufacturing resource heat map and (b) is the manufacturing resource positioning and tracking map.

\section{Conclusion}

Based on the wireless data acquisition terminal in the intelligent workshop, this paper established a resource positioning perceptual model. The architectural structure of real-time resource positioning system was designed based on WSN, and the resource positioning flow was illustrated. To improve the positioning accuracy of the system, a multi-sensor positioning data fusion algorithm was proposed. Finally, the position system was developed. In the following work, how to use resource data to monitor and analyze the manufacturing process and provide reference for decisionmakers will need to be further study.

\section{$7 \quad$ Acknowledgment}

This work was supported by national Natural Science Foundation of China (51475097 and 61540066), Science and Technology Foundation of Guizhou Province (JZ[2014]2001), and National Key Technology Support Program of China (2012BAH62F00). 


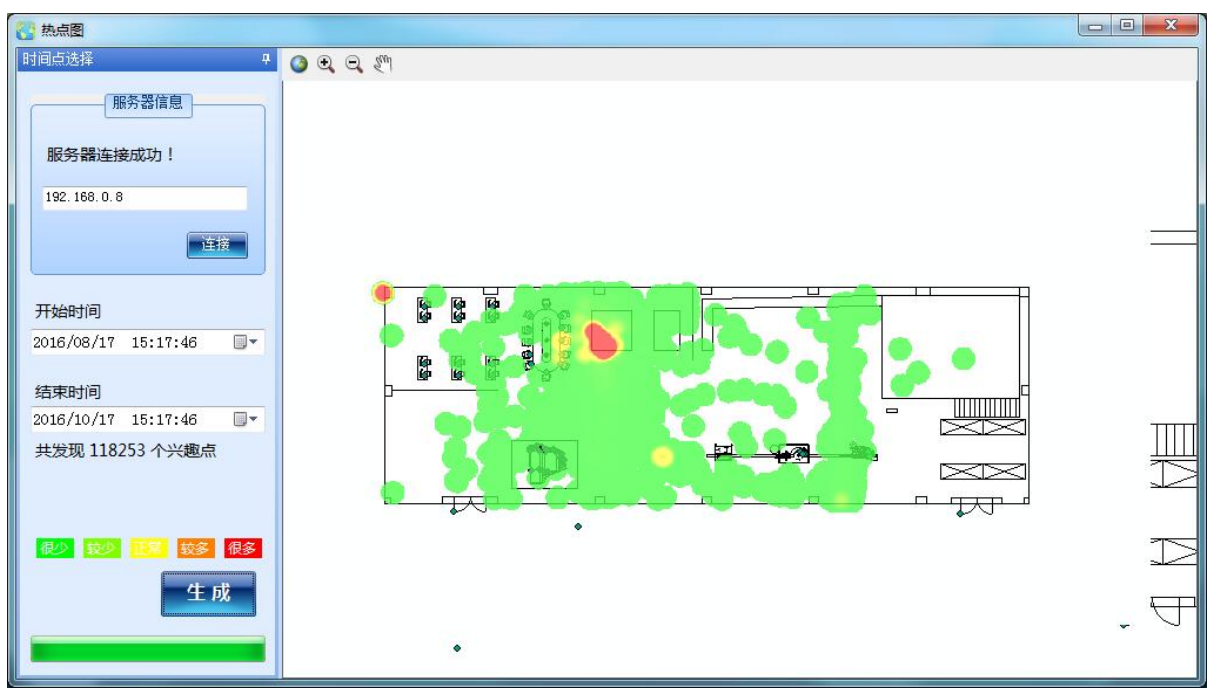

(a) Manufacturing resource heat map

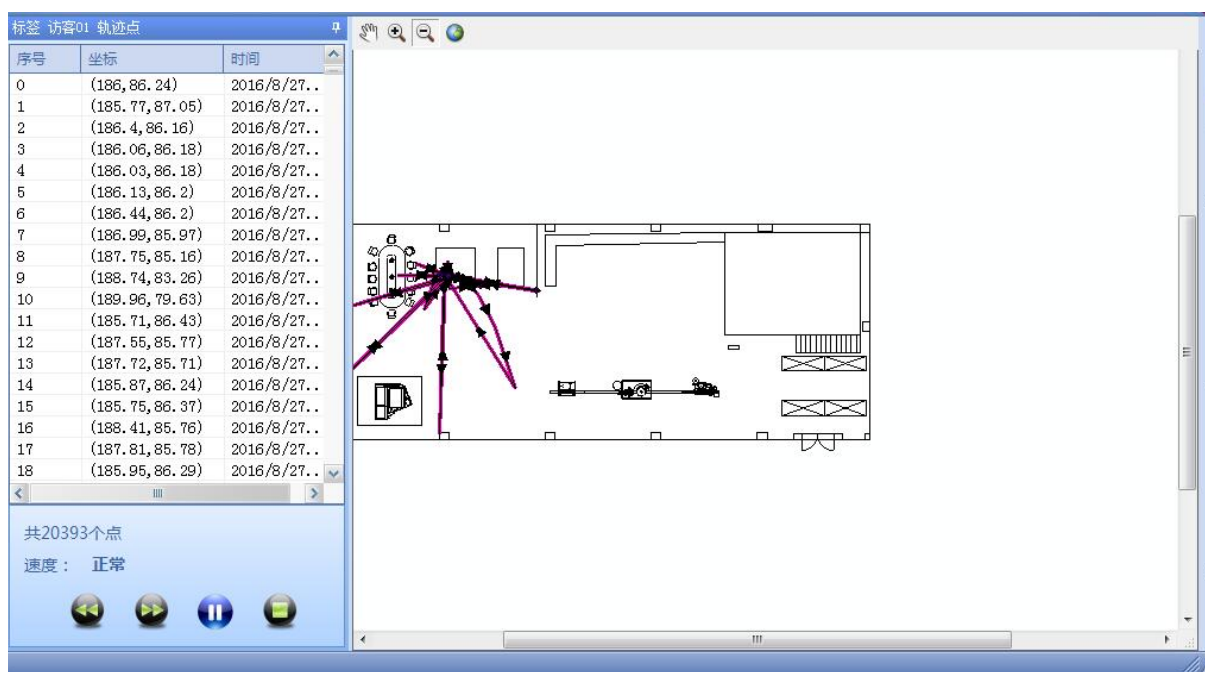

(b) Resource positioning and tracking map

Fig. 4. Part of the system application interface

\section{References}

[1] Vamvoudakis K.G., Jagannathan S. Recent advances and future directions on adaptation and control workshop. IEEE Control Systems, vol. 36, no. 2, pp. 128-130, 2016. https://doi.org/10.1109/MCS.2015.2512083. 
[2] Chen Weixing, Li Shaobo, Huang Haisong. Active perception and management model for manufacturing data in discrete IoMT-based process. Computer Integrated Manufacturing Systems, vol. 22, no.1, pp. 166-176, 2016. https://doi.org/10.13196/j.cims.2016.01.016

[3] Pietro Gonizzi, Gianluigi Ferrari, Vincent Gay, and Jérémie Leguay, Data dissemination scheme for distributed storage for IoT observation systems at large scale. Information Fusion, vol. 22, pp. 16-25, 2015. https://doi.org/10.1016/j.inffus.2013.04.003

[4] Asato Osvaldo L., Pessoa Marcosiris A. O., Junqueira Fabrício. Using the enhanced-mark flow graph for dynamic resource allocation in distributed manufacturing. International Journal of Computer Integrated Manufacturing, vol. 29, no. 11, pp. 1238-1251, 2016. https://doi.org/10.1080/0951192X.2015.1126000

[5] Su Kaikai, Xu Wensheng, Li Jianyong. Manufacturing resource allocation method based on bi-level programming in cloud manufacturing. Computer Integrated Manufacturing Systems, vol. 21, no. 7, pp. 166-176, 2016. https://doi.org/10.13196/j.cims.2016.01.016

[6] Hou Liqun, Yang Lei. Design and implementation of an industrial wireless sensor network for temperature monitoring. International Journal of Online Engineering, vol. 12, no. 3, pp. 82-85, 2016. https://doi.org/10.3991/ijoe.v12i03.5322

[7] Hua Li, Da Xu, Jian Zhang. Design of a state monitoring system for equipment based on the Zigbee wireless sensor network. International Journal of Online Engineering, vol. 12, no. 6, pp. 20-23, 2016. https://doi.org/10.3991/ijoe.v12i06.5516

[8] Yu Xiaoqing, Han Wengting, Zhang Zenglin. Remote monitoring system for intelligent irrigation in hybrid wireless sensor networks. International Journal of Control and Automation, vol. 8, no. 3, pp. 185-196, 2015. https://doi.org/10.14257/ijca.2015.8.3.20

[9] Zhang Lieping, Peng Fei, Cao Peng. An improved three-dimensional DV-Hop localization algorithm optimized by adaptive cuckoo search algorithm. International Journal of Online Engineering, vol. 13, no. 2, pp. 102-118, 2017. https://doi.org/10.3991/ijoe. v13i02.6358

\section{Authors}

Shaobo Li is currently a professor of School of Mechanical Engineering at Guizhou Unversity, Guiyang, China, 550025. His research interests include computational intelligence, manufacturing information system and internet things of technology. $\mathrm{He}$ is a member of the IEEE, CMES and CCF (lishaobo@gzu.edu.cn).

Jinglei Qu is now pursuing his doctor degree in Chengdu Institute of Computer Application at Chinese Academy of Sciences, Chengdu, China, 550025. His research interests include computational intelligence, manufacturing information system and data mining (jinglei0526@163.com).

Chenglong Zhang is now pursuing his master degree in college of big data and information engineering at Guizhou University, Guiyang, China, 550025. His research interests include wireless sensor network (995010174@qq.com).

Article submitted 20 March 2017. Published as resubmitted by the authors 05 May 2017. 